\title{
Role of Surveillance in Prevention of Hepatocellular Carcinoma
}

\author{
Dipanjan Panda \\ Department of Medical Oncology, Institute of Liver and Biliary Sciences, D1 Vasant Kunj, New Delhi 110014, India
}

\begin{abstract}
Hepatocellular carcinoma is a common malignancy and one of the important public health problems in India. The surveillance of hepatocellular carcinoma (HCC) is an established approach to detect early cancers in patients with defined risks. However, there are still controversies and issues to be addressed regarding the optimal surveillance methods and interval. The current level of awareness among physicians in India about surveillance is low and the need and most cost effective surveillance strategy in developing country like ours is unclear. This article has tried to discuss these issues in their appropriate perspective. To address this complicated issue, a multicenter randomized prospective study however may be required. (J Clin Exp HePATOL 2014;4:S43-S49)
\end{abstract}

$\mathrm{H}$ epatocellular carcinoma (HCC) is the fifth commonest cancer worldwide and third commonest cause of cancer related mortality. ${ }^{1}$ In India, the mean incidence of HCC as per four population-based registries was $2.77 \%$ for males and $1.38 \%$ for females. The prevalence of HCC in India varies from $0.2 \%$ to $1.6 \% .{ }^{2,3}$ It is interesting to note that the consolidated data from cancer registries of India do not reflect HCC as an important malignancy. ${ }^{4}$

Risk of HCC development is high in patients with liver cirrhosis of any etiology, particularly with chronic hepatitis $B$ virus (HBV) or hepatitis C virus (HCV) infection. ${ }^{5}$ Since India has a high prevalence of HBV infection (4\% of population, i.e about 40 million Indians) ${ }^{6}$ as well as HCV infection $(0.3-1.5 \%)$ it could contribute to the high incidence of HCC. ${ }^{7}$ Other risk factors for HCC like high prevalence of Nonalcoholic Fatty Liver Disease (NAFLD) obesity, diabetes, alcohol consumption among males are also present in India.

Nonalcoholic Fatty Liver Disease (NAFLD) particularly its more aggressive form, nonalcoholic steatohepatitis (NASH) is an important factor which can lead to cirrhosis and HCC. It is estimated that nearly two-thirds of obese

\footnotetext{
Keywords: surveillance, hepatocellular carcinoma, prevention, cost effective strategy

Received: 10.9.2013; Accepted: 3.3.2014; Available online 25.5.2014

Address for correspondence: Dipanjan Panda, Department of Medical Oncology, Institute of Liver and Biliary Sciences, D1 Vasant Kunj, New Delhi 110014, India. Tel.: +919540946934

E-mails: dipanjan.panda@gmail.com; somdattap@gmail.com

Abbreviations: AFP: alpha-fetoprotein; CI: confidence interval; CLD: chronic liver disease; DCP: des- $\gamma$-carboxy-prothrombin; HBV: hepatitis B virus; HCC: hepatocellular carcinoma; HCV: hepatitis C virus; NAFLD: Nonalcoholic Fatty Liver Disease; NASH: nonalcoholic steatohepatitis; QI: quality improvement; RCT: randomized controlled trial; TPCT: triple phase CT scan; US: ultrasound; USG: ultrasonography; USG: ultra sonogram

http://dx.doi.org/10.1016/j.jceh.2014.03.044
}

people have some form of fatty liver, ranging from steatosis to NASH. NASH can progress to liver cirrhosis in 3\%-15\% and subsequently to liver cancer. ${ }^{8}$ A study by Prasad et al showed that age-standardized prevalence rates of metabolic syndrome were $33.5 \%$ overall, $24.9 \%$ in males and $42.3 \%$ in females in India. Authors concluded that metabolic syndrome is a significant public health problem even in one of the poorest states of India. ${ }^{9}$ Hence NASH is particularly important in countries like India where metabolic syndrome is on the rise.

It is important to note that in the absence of an effective screening program and only $7 \%$ of population being covered by cancer registries, under reporting cannot be ruled out. ${ }^{10}$ A recent study in which 130 trained physicians independently assigned causes to 122429 deaths, which occurred in 1.1 million homes in 6671 small areas that were randomly selected to be representative of all of India, based on a structured nonmedical surveyor's field report has put the liver cancer as the 4th commonest cause of death in male and 8th commonest cause of death in female. ${ }^{11}$

Survival in HCC directly correlates with the stage of the disease. In early stage patients who receive potentially curative therapy, a considerable improvement in survival has been observed (5-year survival ranges between $40 \%$ and $70 \%)$ as compared to the patients who present late (5year survival less than $5 \%$ ). However, the treatments are received by only a small number of patients. Data from developed country like United States based on population-based studies indicate that only approximately $10 \%$ of patients with HCC receive these treatments. ${ }^{12}$ Against this background, surveillance plays a key role. HCC surveillance has been advocated to detect HCC at an early stage, when critical treatment can be applied and hence higher survival can be expected.

A successful surveillance strategy if in place will be able to detect and prevent premature deaths from HCC. In a study from Japan, $81 \%$ of HCC detected by surveillance 
$(n=391)$ were considered suitable for curative resection compared with $46 \%$ of symptomatic HCC $(n=1251) .{ }^{13}$ The overall 5 -year survival rate was $51 \%$ for asymptomatic tumors compared to $5-21 \%$ for symptomatic disease. But cost-effectiveness of surveillance still remained a controversial issue in resource poor developing countries like India.

There is no randomized controlled trial for HCC surveillance although this was previously attempted. In a study to test the feasibility of conducting a randomized controlled trial (RCT) of HCC surveillance in patients with cirrhosis authors concluded that although an RCT is theoretically ideal for determining the efficacy, efficiency, and cost-effectiveness of HCC screening, informed patients prefer surveillance. A randomized study of HCC screening is not feasible when informed consent is imparted. ${ }^{14}$ Hence it would be important for a study to be planned addressing various challenges which would help to understand importance of HCC surveillance.

The aim of this article is to discuss-

- Surveillance and its role in HCC

- Current level of awareness about surveillance among physicians managing CLD patients

- Role of dedicated HCC surveillance program: evidences from different countries

- A cost effective and feasible surveillance strategy in Indian context

- Role of surveillance in post surgery HCC patients.

\section{SURVEILLANCE}

Surveillance consists of periodic application of a diagnostic test (screening test) to subjects at risk for developing a given disease. Screening is traditionally defined as onetime application of a diagnostic test amongst asymptomatic individuals, in order to identify unrecognized early disease or precursors of disease. Several criteria need to be met, for a surveillance program such as 1) disease or condition must be fairly prevalent in the general population/subpopulation, 2) must be an important public health problem, 3) the surveillance test should be safe, free of serious adverse effects, and acceptable to the population being served, 4) the test should be able to diagnose the disease at a stage in which treatment is still possible and which is much more effective than when diagnosed in the nonsurveillance population, 5) benefits gained should reflect in the population level not in the individual level.

The major rationale behind surveillance program for HCC is that patients diagnosed with HCC during surveillance have smaller tumors which are more often amenable to treatment and they can live longer than patients diagnosed with HCC during routine clinical care. ${ }^{15}$ Surveillance is also possible because of existence of a well defined high risk group.

The tests most commonly used for surveillance of HCC are measurement of tumor marker alfa-fetoprotein (AFP) and or ultrasonography (USG), both of which are widely acceptable in general population. Ultrasound may play an important role in HCC surveillance. A study was conducted by Singal et al (2009) to determine the performance characteristics of surveillance with ultrasound for the detection of HCC, particularly early HCC as defined by the Milan criteria. Based on the literature review, authors concluded that the surveillance ultrasound detected the majority of tumours before they presented clinically, with a pooled sensitivity of $94 \%$. However, ultrasound was less effective for detecting early HCC with a sensitivity of $63 \%$. Alpha-fetoprotein provided no additional benefit to ultrasound. Although surveillance with ultrasound demonstrates limited sensitivity for early HCC, this may be improved by testing at 6-month intervals. ${ }^{16}$ However this study did not address the effectiveness in clinical situation. A study was conducted to evaluate the effectiveness of a surveillance program with ultrasound and alphafetoprotein (AFP) in clinical practice to detect early HCCs. In this prospective study conducted in 446 patients with Child A/B cirrhosis, surveillance ultrasound and AFP had sensitivities of $44 \%$ and $66 \%$ and specificities of $92 \%$ and $91 \%$, respectively, for the detection of HCCs. Sensitivity significantly improved to $90 \%$, with minimal loss in specificity $(83 \%)$ when these tests were used in combination. $^{17}$

In a prospective study conducted in India at All India Institute of Medical Sciences, New Delhi, 194 patients with liver cirrhosis of varied etiology with no detectable HCC were followed up for a mean period of 34.9 months. Serum AFP measurement, abdominal ultra sonogram (USG) was done every 6 months, and triple phase CT scan (TPCT) every year. The authors found that in most patients, liver cirrhosis was related to $\mathrm{HBV}$ infection, $\mathrm{HCV}$ infection or both $(36.6 \%, 27.8 \%$ and $6.2 \%$, respectively). During a cumulative follow-up of 563.4 person-years, HCC was detected in 9 subjects, having an incidence rate of 1.60 (95\% confidence interval $0.55-2.64$ ) per personyear. All had infection with HBV, HCV or both $(4,4$ and 1 , respectively). ${ }^{18}$

A limiting factor for such surveillance programs is the failures and they potentially contribute to late-stage tumors in one-third of cases. The most common reason for finding HCC at a late stage was an absence of detection, suggesting better surveillance strategies are needed. Various studies show significant variation in the effectiveness of surveillance programmes. Variations in physicianand system-level factors are more important than patient-level factors in determining surveillance rates and they may contribute to late stage tumor presentation. ${ }^{19}$

There are several other novel biomarkers which are currently being assessed. Marrero et al, 2009 evaluated des- $\gamma$-carboxy-prothrombin (DCP) and lectin-bound AFP (AFP-L3\%) as potential surveillance tests for HCC. They compared these two biomarkers with AFP and concluded 
that AFP was more sensitive than DCP and AFP-L3\% for the diagnosis of early and very early stage $\mathrm{HCC}^{20}$ In another study, des- $\gamma$-carboxy-prothrombin and alphafetoprotein as biomarkers for the early detection of hepatocellular carcinoma were evaluated. However, neither DCP nor AFP were proven to be optimal. ${ }^{21}$

Problems associated with these surveillance programmes are various biases like lead-time bias (apparent improvement in survival), prognostic selection bias (identification of patients with slow progressive tumors who are more likely to live longer) and over diagnosis bias (false positives). However, there are studies which show a benefit despite statistically adjusting for lead time bias. ${ }^{22,23}$

\section{RECALL PROCEDURES}

Standardized recall procedures which require a standardized plan for subsequent evaluation of patients found to have a positive surveillance test is very important. Although various recall algorithms have been described in the context of liver disease, there is a paucity of literature for tests evaluated rigorously in a prospective fashion. Increases in serum AFP level need to be interpreted against a background of liver disease. Exacerbations of HBV infection, pregnancy which may cause temporary increases in AFP levels need to be considered for raised AFP level. There is requirement of re measuring AFP levels and a requirement to carry out detailed clinical evaluation to determine causes for increases other than HCC. In contrast with AFP, a new mass or hypodense lesion in the liver detected by ultrasound surveillance is very suggestive of HCC and requires complete evaluation. This may be achieved by further imaging by computerized tomography or magnetic resonance imaging. ${ }^{24}$

\section{CURRENT LEVEL OF AWARENESS ABOUT HEPATOCELLULAR CARCINOMA SURVEILLANCE AMONG PHYSICIANS MANAGING CHRONIC LIVER DISEASE (CLD) PATIENTS}

There is currently no available data about the level of awareness among Indian doctors regarding need of surveillance of CLD patients. In an American study conducted to identify HCC patients and obtain data on $n$ patient characteristics, health care providers, and treatment utilization, a health care claims database was used. Data revealed that primary care/internal medicine providers managed the majority of HCC cases (55\%) and oncologist (24\%) managed minority of the patients. The cases known to have cirrhosis who were undergoing HCC screening prior to diagnosis were only $22 \%$. This study identified an existing gap between screening and treatment guidelines and practice patterns, implying a need for greater health care provider awareness and education. ${ }^{25}$ In another study, approximately $48 \%$ of patients were seen by a gastroenterologist/hepatologist or by a physician with an academic affiliation. Patients were approximately 4.5 -fold and 2.8-fold, respectively, more likely to receive regular surveillance than those seen by a primary care physician only. ${ }^{26}$ In a systemic review, surveillance rates were significantly higher among patients followed in subspecialty gastroenterology clinics compared to those followed in primary care clinics $(51.7 \%$ vs. $16.9 \%, p<0.001) .{ }^{27}$ There has been a study conducted to understand failure rates in surveillance. The surveillance was reported to be more likely among patients seen by hepatologists [OR, 6.11; 95\% confidence interval (CI), 2.5-14.8] and less likely in those with alcohol abuse (OR, 0.14; 95\% CI, 0.03-0.65). There were multiple points of failure in the surveillance process, with the most common being failure to order surveillance in patients with known cirrhosis. ${ }^{28}$

Quality improvement can play a key role in improving surveillance. In a study to evaluate the effectiveness of implementing quality improvement (QI) measures in increasing the rate of HCC surveillance among patients in a tertiary care facility, most common reasons for failure to undergo surveillance were reported to be patients' lack of insurance and lack of follow-up on studies ordered at outside institutions. Authors through this study concluded that the implementation of QI measures incorporating automatic reminders of surveillance status for providers can significantly increase the rate of HCC surveillance among cirrhosis patients. ${ }^{29}$

\section{ROLE OF DEDICATED HEPATOCELLULAR CARCINOMA SURVEILLANCE PROGRAM: EVIDENCES FROM DIFFERENT COUNTRIES}

One large population-based study from China has demonstrated survival benefit of a surveillance program in chronic hepatitis B patients. In this study, a randomized controlled trial (RCT), 18816 subjects with history of chronic hepatitis were randomly assigned to 'screening' and 'control' groups (9373 and 9443 subjects respectively). Surveillance was done using 6-monthly AFP and USG and the groups were followed for 38,444 and 41,077 personyears. HCC surveillance group mortality rate was significantly lower $(83.2 / 100,000)$ than the unscreened group $\left(131.5 / 100,000 .^{30}\right.$

Another study from China also an RCT which included 5581 chronic hepatitis B carriers found surveillance was not associated with any reduction in mortality. ${ }^{31}$ This study however included only AFP as a surveillance method without including sonography. This along with the fact that there were low rates of curative therapies despite early stage detection could explain lack of reduction in mortality in this study as compared to the population based study mentioned earlier. $^{30,31}$ 
Data from a systematic review from U. K showed surveillance group to have estimated three times increase in the number of patients with operable HCC tumours at diagnosis, and almost halve the number who dies from HCC compared to non-surveillance group. ${ }^{32}$ In clinic-based surveillance studies in Italy and Taiwan, liver-specific mortality rates were reduced in cirrhotic patients with HCC detected during surveillance. ${ }^{33,34}$ Retrospective studies from Taiwan, Hong Kong and Italy also reported small and potentially curable HCC being detected in the surveillance groups and leading to improved patient survival. ${ }^{35}$

\section{COST-EFFECTIVE AND FEASIBLE STRATEGY FOR HEPATOCELLULAR CARCINOMA SURVEILLANCE PARTICULARLY FOR INDIAN SET-UP?}

Multiple studies in different part of the world have been conducted to find out most cost effective and feasible HCC surveillance strategy. It is important to understand the recommended surveillance interval. In a study when semi-annual and annual surveillance plans were compared, semi-annual and annual surveillance was shown to equally improve the survival of cirrhotic patients with hepatocellular carcinoma and greatly increase the amenability rate to liver transplantation. ${ }^{22}$ In the meta analysis conducted by
Singal et al, studies with surveillance at least every 6 months had a significantly higher sensitivity for early stage HCC than studies performing surveillance on an annual basis $(P=0.001) \cdot{ }^{16}$ However, recently a randomized trial was conducted comparing 3- and 6-month periodicities in Ultrasonographic surveillance of hepatocellular carcinoma in cirrhosis and authors concluded that surveillance, performed every 3 months, detects more small focal lesions than US every 6 months, but does not improve detection of small HCC, probably because of limitations in recall procedures. ${ }^{36}$ A prospective cohort study from Italy which had done surveillance using AFP and USG at 6-month intervals found the cost to be US $\$ 112,993$ per life-year saved. ${ }^{37}$

Two systematic reviews have also looked at cost effective strategies for surveillance in HCC. The first Systematic review by Coon et al had surveillance of patients with cirrhosis (related to Alcoholic Liver Diseases, HBV, HCV infection), using AFP testing and/or liver ultrasound examination, to detect HCC, followed by treatment with liver transplantation or resection. The result showed that surveillance using AFP and USG at 6month intervals would nearly halve the number of HCC-related deaths. Undiscounted lifetime cost of this surveillance strategy was 42,900 British pounds (mostly transplant related cost). ${ }^{32}$ The second systematic review by Ruggeri et al concluded that incidence is the key

Table 1 International Guidelines on Modalities of Surveillance. ${ }^{41-44}$

\begin{tabular}{ll}
\hline Societies & \multicolumn{1}{c}{ Recommendations and (level of evidence/recommendation) } \\
\hline EASL & $\begin{array}{c}\text { Patients at high risk for developing HCC should be entered into surveillance } \\
\text { programs. (1B/3A,1A/B) }\end{array}$ \\
& $\begin{array}{l}\text { - Surveillance should be performed by experienced personnel in all at-risk } \\
\text { populations using abdominal ultrasound every } 6 \text { months }(2 \mathrm{D} ; 1 \mathrm{~B})\end{array}$ \\
SASLD & $\begin{array}{l}\text { Surveillance has to be based on ultrasound examination. The recommended } \\
\text { screening interval is } 6 \text { months. }\end{array}$ \\
ESMO & $\begin{array}{l}\text { Surveillance of patients at risk for HCC should be carried out by abdominal } \\
\text { ultrasound every } 6 \text { months }[1, \mathrm{~A}]\end{array}$
\end{tabular}

Levels of evidence according to study design and end-points ${ }^{45}$

- National Cancer Institute: PDQ levels of evidence for adult and pediatric cancer treatment studies.

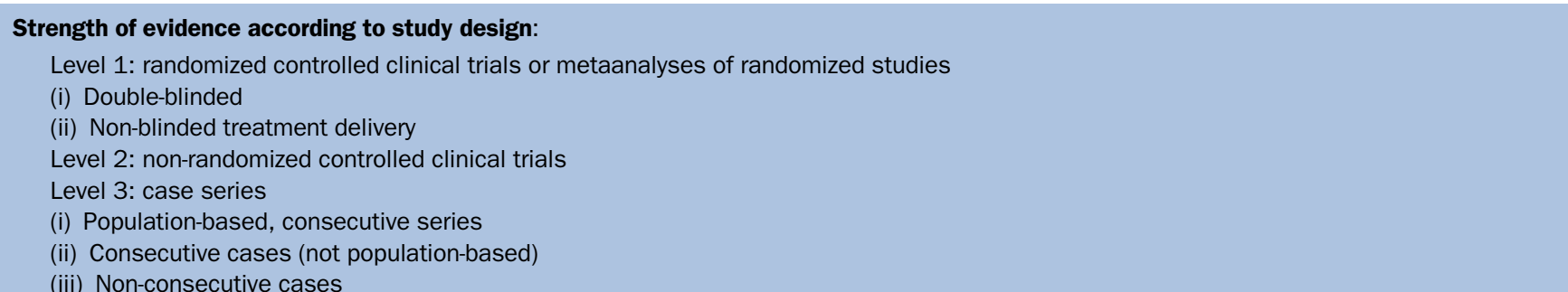

\section{Strength of evidence according to end-points:}

A. Total mortality (or overall survival from a defined time)

B. Cause-specific mortality (or cause-specific mortality from a defined time)

C. Carefully assessed quality of life

D. Indirect surrogates
(i) Event-free survival
(ii) Disease-free survival
(iii) Progression-free survival
(iv) Tumor response rate 
parameter which determines the cost-effectiveness of HCC screening and 6 monthly surveillance was shown to be the most cost effective timing strategy. Regarding modalities of surveillance the authors concluded that USG alone or in association with AFP technology is likely to be the most cost effective and use of CT shows controversial results. The review also concluded that screening should be implemented to detect HCC at an early stage of cirrhosis and it is likely to be not cost effective as liver cirrhosis progresses or after liver transplantation. ${ }^{38}$

Another multicenter retrospective study from Italy on 1051 consecutive patients with hepatocellular carcinoma suggested that surveillance should be offered only if the risk of HCC in a particular group exceeds $1.5 \%$ per year. ${ }^{22}$

One RCTs have shown that absolute reduction in annual HCC-related mortality rate was 48.3 deaths per 100,000 populations ${ }^{31}$ which implies that More than 2000 person-years of surveillance would be needed to prevent one death due to HCC. ${ }^{39}$ Cost effective surveillance strategy in Indian population was assessed in a Prospective study undertaken to estimate the incidence of HCC in 194 cirrhotics who underwent 6 monthly abdominal ultrasound (US) and serum AFP) estimation, and yearly TPCT. Cost was estimated from the hospital and patient perspectives. Cost-effectiveness ratios for detecting a case of HCC were estimated. Modeling was done to estimate cost-effectiveness with different combinations of diagnostic tests. Result of the study showed cost-effectiveness ratios of HCC surveillance program per HCC case detected were estimated as US\$280 (hospital perspective). From patient perspective the cost was US\$ 9965 for outstation patient and US\$ 2808 for local patients. Cost-effectiveness ratio for direct medical cost per case of HCC detected by 6 monthly US and AFP, the EASL protocol, was estimated to be US\$ 1510 in the private sector. ${ }^{40}$

Most of the international societies recommend use of USG only as cost effective tools for surveillance of HCC (Table 1). ${ }^{41-44}$

\section{SURVEILLANCE STRATEGY POST SUCCESSFUL TREATMENT FOR HEPATOCELLULAR CARCINOMA?}

The overall rate of recurrence of hepatocellular carcinoma (HCC), after liver transplantation ranged from 10 to $20 \%$ with some differences in pre-transplant selection criteria. $^{45-48}$ If explant pathology is within the currently accepted criteria for transplantation then rate of recurrence is low (8\%) but on the other hand If pathology is outside of the criteria then chance of recurrence is as high as $50 \% .{ }^{45}$ In two of above mentioned studies majority of patients had recurrence within 12 and 18 months respectively. As time to recurrence is early, it seems if screening is effective, it should be done in immediate post-operative period.

\section{MODALITIES OF SURVEILLANCE TO DETECT POST-TRANSPLANT RECURRENCE}

There is no established surveillance protocol for recurrent HCC following liver transplantation. Hence many recommendations may be based on the expert opinion instead of well conducted studies. In a study conducted by regalia et al HCC recurrence was detected in $71 \%$ patients within 18 months after orthotopic liver transplantation. ${ }^{47}$ A Japanese study has suggested that frequent measurement of AFP and des- $\gamma$-carboxy-prothrombin was useful for detecting recurrent HCC. This may be useful for long-term follow-up markers for post-transplant surveillance. ${ }^{49}$

\section{COST OF POST-TRANSPLANT SURVEILLANCE}

Treatment is curative in only about $1 \%$ of those with HCC recurrence after liver transplantation. Cost of screening in western country is estimated about 100000 USD (screening on a 6-month basis with CT scans and AFP). Screening only patients with explant pathology outside of the Milan criteria would result in a smaller number of patients screened, presumably with the same rate of cure. ${ }^{50}$

\section{CONCLUSION}

HCC surveillance can detect early tumors that are potentially amendable to curative treatment. All patients at risk of developing HCC for which potential curative treatment available are candidate for regular HCC surveillance. Presently there is very limited data regarding level of awareness and attitude of physicians managing CLD patients which is a major factor in surveillance indicating that there is a need for health care provider awareness and education.

However, it is important to note that as a secondary prevention strategy surveillance is economically viable only for a selected group of patients and may not be viable in resource constraint country like India. Regarding surveillance strategy in post-transplant patients semi-annual imaging for first two years may be successful in our country. Although challenging to perform, a large multicentre prospective trial may be required to shed more light in this controversial issue.

\section{CONFLICTS OF INTEREST}

The author has none to declare.

\section{REFERENCES}

1. Tsukuma H, Hiyama T, Tanaka S, et al. Risk factors for hepatocelIular carcinoma among patients with chronic liver disease. N Eng/ J Med. 1993 24;328(25):1797-1801.

2. Annual Report 1987-1990. National Cancer Registry Programme. New Delhi: Indian Council of Medical Research. 
3. Jayant K, Rao RS, Nene BM, Dale PS, Barshi. Rural Cancer Registry. 1994. Rural Cancer Registry at Barshi-Report 1988-92.

4. National Cancer Registry Program: Consolidated Report of Population Based Cancer Registries 2001-2004. Indian Council of Medical Research Publication http://www.icmr.nic.in/ncrp/report_ pop_2001-04 as Accessed 15.01.14.

5. Beasley RP, Hwang LY, Lin CC, Chien CS. Hepatocellular carcinoma and hepatitis B virus. A prospective study of 22707 men in Taiwan. Lancet. 1981 Nov 21;2(8256):1129-1133.

6. Tandon BN, Acharya SK, Tandon A. Epidemiology of hepatitis B virus infection in India. Gut. 1996;38(suppl 2):S56-S59.

7. Chowdhury A, Santra A, Chaudhuri S, et al. Hepatitis C virus infection in the general population: a community-based study in West Bengal, India. Hepatology. 2003 Apr;37(4):802-809.

8. Ascha MS, Hanouneh IA, Lopez R, Tamimi TA, Feldstein AF, Zein NN. The incidence and risk factors of hepatocellular carcinoma in patients with nonalcoholic steatohepatitis. Hepatology. 2010;51:1972-1978.

9. Prasad DS, Kabir Z, Dash AK, Das BC. Prevalence and risk factors for metabolic syndrome in Asian Indians: a community study from urban eastern India. J Cardiovasc Dis Res. 2012 JulSep;3(3):204-211.

10. Acharya SK, Madan K, Dattagupta S, Panda PK. Viral hepatitis in India. Natl Med J India. 2006;19(4) http://www.nmji.in/archives/ volume_19_4_Jul_Aug_2006/SPECIAL_SERIES/Special_Series_ 19_4.htm. as Accessed 15.01.14.

11. Dikshit R, Gupta PC, Ramasundarahettige C, et al. Cancer mortality in India: a nationally representative survey. Lancet. 2012;379(9828):1807-1816.

12. El-Serag Hashem B, Davila Jessica A. Surveillance for hepatocelluIar carcinoma: in whom and how? Ther Adv Gastroenterol. 2011 January;4(1):5-10.

13. Tang ZY, Yu YQ, Zhou XD, Yang BH, Ma ZC, Lin ZY. Subclinical hepatocellular carcinoma: an analysis of 391 patients. J Surg Oncol Suppl. 1993;3:55-58.

14. Poustchi H, Farrell GC, Strasser SI, Lee AU, McCaughan GW, George J. Feasibility of conducting a randomized control trial for liver cancer screening: is a randomized controlled trial for liver cancer screening feasible or still needed? Hepatology. 2011 Dec;54(6):1998-2004.

15. Collier J, Sherman M. Screening for hepatocellular carcinoma. Hepatology. 1998 Jan;27(1):273-278.

16. Singal A, Volk ML, Waljee A, et al. Meta-analysis: surveillance with ultrasound for early-stage hepatocellular carcinoma in patients with cirrhosis. Aliment Pharmacol Ther. 2009;30:37-47.

17. Singal AG, Conjeevaram HS, Volk ML, et al. Effectiveness of hepatocellular carcinoma surveillance in patients with cirrhosis. Cancer Epidemiol Biomarkers Prev. 2012;21(5):793-799.

18. Paul SB, Sreenivas V, Gulati MS, et al. Incidence of hepatocellular carcinoma among Indian patients with cirrhosis of liver: an experience from a tertiary care center in northern India. Indian J Gastroenterol. 2007 Nov-Dec;26(6):274-278.

19. Singal AG, Nehra M, Adams-Huet B, et al. Detection of hepatocellular carcinoma at advanced stages among patients in the HALT-C trial: where did surveillance fail? Am J Gastroenterol. 2013;108:425-432.

20. Marrero JA, Feng Z, Wang Y, et al. $\alpha$-Fetoprotein, Des- $\gamma$ carboxyprothrombin, and lectin-bound $\alpha$-fetoprotein in early hepatocellular carcinoma. Gastroenterology. 2009;137(1):110-118.

21. Lok AS, Sterling RK, Everhart JE, et al, the HALT-C Trial Group. Desgamma-Carboxy prothrombin and alpha-fetoprotein as biomarkers for the early detection of hepatocellular carcinoma. Gastroenterology. 2010 Feb;138(2):493-502.

22. Trevisani F, De NS, Rapaccini G, et al. Semiannual and annual surveillance of cirrhotic patients for hepatocellular carcinoma: effects on cancer stage and patient survival (Italian experience). Am J Gastroenterol. 2002;97:734-744.

23. Tanaka N, Ichijo T, Okiyama W, et al. Laparoscopic findings in patients with nonalcoholic steatohepatitis. Liver Int. 2006;26:32-38.

24. Bisceglie AD. Issues in screening and surveillance for hepatocellular carcinoma. Gastroenterology. 2004;127:S104-S107.

25. Sanyal A, Poklepovic A, Moyneur E, Barghout V. Population-based risk factors and resource utilization for HCC: US perspective. Curr Med Res Opin. 2010 Sep;26(9):2183-2191.

26. Davila JA, Morgan RO, Richardson PA, Du XL, McGlynn KA, ElSerag HB. Use of surveillance for hepatocellular carcinoma among patients with cirrhosis in the United States. Hepatology. 2010 Jul;52(1):132-141.

27. Singal AG, Yopp A. Utilization of hepatocellular carcinoma surveillance among American patients: a systematic review. J Gen Intern Med. 2012; Jul;27(7):861-867.

28. Singal AG, Yopp AC, Gupta S, et al. Failure rates in the hepatocellular carcinoma surveillance process. Cancer Prev Res (Phila). 2012;5(9):1124-1130.

29. Aberra FB, Essenmacher M, Fisher N, Volk ML. Quality improvement measures Lead to higher surveillance rates for hepatocellular carcinoma in patients with cirrhosis. Dig Dis Sci. 2013 Apr;58(4):1157-1160.

30. Zhang BH, Yang BH, Tang ZY. Randomized controlled trial of screening for hepatocellular carcinoma. J Cancer Res Clin Oncol. 2004 Jul;130(7):417-422.

31. Chen JG, Parkin DM, Chen QG, et al. Screening for liver cancer: results of a RCT in Qidong. China J Med Screen. 2003;10:204209.

32. Thompson Coon J, Rogers G, Hewson P, et al. Surveillance of cirrhosis for hepatocellular carcinoma: systematic review and economic analysis. Health Technol Assess. 2007 Sep;11(34):1-206.

33. Sangiovanni A, Del Ninno E, Fasani P, et al. Increased survival of cirrhotic patients with a hepatocellular carcinoma detected during surveillance. Gastroenterology. 2004 Apr;126(4):1005-1014.

34. Chie WC, Chang YH, Chen HH. A novel method for evaluation of improved survival trend for common cancer: early detection or improvement of medical care. J Eval Clin Pract. 2007 Feb;13(1):79-85.

35. Amarapurkar D, Han KH, Chan HL, Ueno Y. Asia-Pacific Working Party on prevention of hepatocellular carcinoma application of surveillance programs for hepatocellular carcinoma in the Asia-Pacific region. J Gastroenterol Hepatol. 2009 Jun;24(6):955-961.

36. Trinchet JC, Chaffaut C, Bourcier V, et al, Groupe d'Etude et de Traitement du Carcinome Hépatocellulaire (GRETCH). Ultrasonographic surveillance of hepatocellular carcinoma in cirrhosis: a randomized trial comparing 3- and 6-month periodicities. Hepatology. 2011 Dec;54(6):1987-1997.

37. Sarasin FP, Giostra E, Hadengue A. Cost-effectiveness of screening for detection of small hepatocellular carcinoma in western patients with Child-Pugh class A cirrhosis. Am J Med. 1996 Oct;101(4):422-434.

38. Ruggeri M. Hepatocellular carcinoma: cost-effectiveness of screening. A systematic review. Risk Manag Healthc Policy. 2012;5:49-54.

39. Aggarwal R. Surveillance for hepatocellular carcinoma in patients with liver cirrhosis in India. Trop Gastroenterol. 2008 Oct Dec;29(4):183-186.

40. Paul SB, Sreenivas V, Gulati MS, et al. Economic evaluation of a surveillance program of hepatocellular carcinoma (HCC) in India. Hepatol Int. 2008 Jun;2(2):231-236.

41. EASL-EORTC clinical practice guidelines: management of hepatocellular carcinoma. J Hepatol. 2012;56:908-943.

42. Bruix J, Sherman M. Management of hepatocellular carcinoma: an update. Hepatology. 2011;53(3):1-35. 
43. Verslype C, Rosmorduc O, Rougier P, on behalf of the ESMO Guidelines Working Group. Hepatocellular carcinoma: ESMO-ESDO clinical practice guidelines for diagnosis, treatment and follow-up. Ann Oncol. 2012;23(suppl 7):vii41-vii48.

44. Mazzaferro V, Regalia E, Doci R, et al. Liver transplantation for the treatment of small hepatocellular carcinomas in patients with cirrhosis. N Engl J Med. 1996;334:693-699.

45. National Cancer Institute. $P D Q^{\circledR}$ Levels of Evidence for Adult and Pediatric cancer Treatment Studies. Bethesda, MD: National Cancer Institute; 2011. Date last modified 26/August/2010 <http:// cancer.gov/cancertopics/pdq/levels-evidence-adult-treatment/ healthprofessional/>. as Accessed 03.03.14.

46. Todo S, Furukawa H, Japanese Study Group on Organ Transplantation. Living donor liver transplantation for adult patients with hepatocellular carcinoma: experience in Japan. Ann Surg. 2004;240:451461.
47. Regalia E, Fassati LR, Valente U, et al. Pattern and management of recurrent hepatocellular carcinoma after liver transplantation. J Hepatobiliary Pancreat Surg. 1998;5:29-34.

48. Roayaie S, Schwartz JD, Sung MW, et al. Recurrence of hepatocelIular carcinoma after liver transplant: patterns and prognosis. Liver Transpl. 2004;10:534-540.

49. Yamashiki N, Sugawara Y, Tamura S, et al. Postoperative surveillance with monthly serum tumor markers after living-donor liver transplantation for hepatocellular carcinoma. Hepatol Res. 2010 Apr;40(4):278-286.

50. Roberts John P. Tumor surveillance-what can and should be done? Screening for recurrence of hepatocellular carcinoma after liver transplantation. Liver Transpl. 2005;11(11):S45S46. 